\title{
Relaçóes entre as estratégias de aprendizagem e motivaçáo no Ensino Técnico Profissionalizante
}

\author{
Adriana Satico Ferraz ${ }^{1}$, Carla Priscila da Silva Pereira ${ }^{2}$, \\ Acácia Aparecida Angeli dos Santos ${ }^{3}$ \\ Universidade São Francisco-Brasil y Faculdade São Leopoldo Mandic-Brasil
}

\begin{abstract}
O principal objetivo desta pesquisa foi investigar a evidência de validade baseada na relação com outras variáveis da Escala de Avaliação de Estratégias de Aprendizagem para o Ensino Profissionalizante. Participaram do estudo 401 estudantes brasileiros. As estratégias de aprendizagem se associaram às metas de realização em nível correlacional e preditivo. $\mathrm{O}$ objetivo secundário foi analisar as diferenciaçóes em ambos os construtos quanto à idade, pausa nos estudos e quantidade de módulos cursados. Os estudantes mais velhos se diferenciaram dos mais jovens na ausência de estratégias metacognitivas disfuncionais e nas estratégias cognitivas. A meta performance-aproximação foi identificada nos estudantes mais jovens e naqueles que fizeram uma pausa intermediária nos estudos. Recomenda-se a continuidade das investigaçôes sobre essa temática.
\end{abstract}

Palavras-chave: métodos de estudo, metas de realização, avaliação psicoeducacional.

Relaciones entre estrategias de aprendizaje y motivación en la Educación Técnica Vocacional El objetivo principal de esta investigación fue investigar la evidencia de validez de una escala que evalúa las estrategias de aprendizaje. Participaron 401 estudiantes brasileños de educación técnica vocacional. Las estrategias de aprendizaje se asociaron con los objetivos de logro - nivel correlacional y predictivo. El objetivo secundario fue analizar las diferencias en ambos constructos en cuanto a edad, pausa en los estudios y número de módulos cursados. Los estudiantes mayores se diferenciaron de los estudiantes más jóvenes en la ausencia de estrategias metacognitivas disfuncionales y estrategias cognitivas. La meta de aproximación al desempeńo se identificó en los estudiantes más jóvenes y en los que tomaron un descanso intermedio de los estudios. Se recomienda realizar más investigaciones sobre este tema. Palabras clave: métodos de estudio, objetivos de logro, evaluación psicoeducativa.

1 Mestre em Psicologia. Doutoranda em Psicologia na Universidade São Francisco, Campinas-SP, Brasil. Endereço postal: Rua José Franco de Oliveira, no 99, Bairro Arraial, Tuiuti-SP, CEP 12930-000, Brasil. Contato: adrianasatico.as@gmail.com https://orcid. org/0000-0002-9856-0094

2 Mestre em Psicologia pela Universidade São Francisco, Campinas-SP, Brasil. Endereço postal: Rua Maria Mercedes Etter Von Zuben, no 151, bloco F, apto 408, Campinas-SP, CEP 13044-503, Campinas-SP, Brasil. Contato: cpsico78@gmail.com https://orcid. org/0000-0003-4911-3471

3 Doutora em Psicologia pela Universidade de São Paulo-SP, Brasil. Mestre em Psicologia Clínica pela Pontifícia Universidade Católica de Campinas-SP, Brasil. Endereço postal: Rua Dom Bosco, 135 ap Taquaral, CEP 13075-060, Campinas-SP. Contato: acacia.santos@ usf.edu.br https://orcid.org/0000-0002-8599-7465 


\section{Relationships between Learning and Motivation Strategies in Vocational Technical Education}

This research's main objective was to investigate the evidence of validity based on the relationship with other variables of the Learning Strategies Assessment Scale for Vocational Education. The sample study was 401 Brazilian students. Learning strategies were associated with achievement goals at a correlational and predictive level. The secondary objective was to analyze the differences in both constructs regarding age, pause in studies, and the number of modules taken. Older students differed from younger students in the absence of dysfunctional metacognitive strategies and cognitive strategies. We identified the performance-approach goal in the younger students and those who took an intermediate break from studies. We recommend further research on this topic.

Keywords: study methods, achievement goals, psychoeducational assessment.

\section{Relations entre les Stratégies d'apprentissage et de Motivation dans L'enseignement Technique Professionnel}

L'objectif principal de cette recherche était d'étudier les preuves de validité basées sur la relation avec d'autres variables de l'échelle d'évaluation des stratégies d'apprentissage pour l'enseignement professionnel. L'échantillon d'étude était de 401 étudiants brésiliens. Les stratégies d'apprentissage étaient associées aux objectifs de réalisation au niveau corrélationnel et prédictif. L'objectif secondaire était d'analyser les différences entre les deux construits concernant l'âge, la pause dans les études et le nombre de modules suivis. Les élèves plus âgés différaient des élèves plus jeunes en l'absence de stratégies métacognitives et cognitives dysfonctionnelles. Nous avons identifié l'objectif de l'approche performance chez les élèves plus jeunes et ceux qui ont pris une pause intermédiaire des études. Nous recommandons de poursuivre les recherches sur ce sujet.

Mots-clés: méthodes d'étude, objectifs de réalisation, évaluation psychoéducative. 
O Ensino Técnico Profissionalizante (ETP) centraliza-se na capacitação para o mercado de trabalho e na inserçáo social. Esse tipo de formaçáo prevê a articulação dos aspectos cognitivos, psicomotores e socioafetivos no desenvolvimento de competências que conferem maiores chances de os estudantes serem bem-sucedidos no exercício da profissão. Dentre as habilidades adquiridas ao longo do curso se destacam a capacidade de analisar as situaçóes, de sintetizar as informações, bem como de estabelecer relaçóes e generalizaçôes (Brasil, 2000; Fedre \& Vitorino, 2015).

Uma forma de otimizar a rotina de estudos dos alunos do ETP é introduzir o uso de estratégias de aprendizagem. No nível cognitivo, as estratégias de aprendizagem fomentam o entendimento da informação, auxiliando na aquisição dos conceitos das aulas. No âmbito metacognitivo, essas estratégias proporcionam uma compreensão mais aprofundada do significado das aulas, percebida no estabelecimento de relaçóes entre a teoria e a prática profissional (Rozendaal, Minnaert, \& Boekaerts, 2003; Scacchetti, Oliveira, \& Moreira, 2015; Slaats, Lodewijks, \& van der Sanden, 1999).

É identificado nos estudantes dessa modalidade de ensino uma ênfase na utilização das estratégias cognitivas, uma vez que o curso é fundamentado em atividades práticas que exigem o desenvolvimento de açóes voltadas para a repetição de procedimentos, para a releitura e para a síntese do conteúdo. Em vista disso, é recomendado que os estudantes também sejam estimulados a desenvolver as estratégias metacognitivas como uma forma de auxiliá-los a se posicionarem criticamente, a reconhecerem os aspectos que podem ser melhorados e a adquirirem habilidades para aprenderem de forma mais autônoma (Scacchetti et al., 2015).

O propósito deste estudo é dar continuidade à investigação sobre o uso das estratégias de aprendizagem, por parte dos estudantes do ETP, 
tendo como objetivo principal analisar a evidência de validade baseada na relação com outras variáveis da Escala de Avaliação de Estratégias de Aprendizagem para o Ensino Profissionalizante (EAVAP-EP; Pereira $\&$ Santos, 2016). Com esta pesquisa pretende-se demonstrar o quanto as estratégias de aprendizagem cognitivas, centralizadas no ensaio, na elaboração e na organização, e as estratégias metacognitivas, com ênfase para o planejamento, a organização, o monitoramento e a regulação podem impactar na motivação dos alunos (Jossberger et al., 2019; Scacchetti, Oliveira, \& Rufini, 2014; Slaats et al., 1999; van Grinsven, \& Tillema, 2006).

Neste empreendimento, o construto motivacional relacionado à EAVAP-EP diz respeito às metas de realização. Essas metas se referem aos propósitos pessoais que direcionam as açóes dos alunos para aquilo que almejam alcançar com os estudos (Bardach et al., 2019). As metas aqui investigadas se referem à meta aprender e às metas performances aproximação e evitação (Bzuneck \& Boruchovitch, 2019).

$\mathrm{Na}$ meta aprender, a motivação é sustentada pelo anseio de elevar o conhecimento intelectual apoiado na dedicação ao curso. Nas metas performances, o foco dos estudantes é o desempenho. A orientação motivacional pela meta performance aproximação é associada ao cumprimento das demandas do curso sob a necessidade do reconhecimento pelo bom desempenho. Por sua vez, na meta performance evitação, se evidencia o receio da exposição em decorrência do fracasso, o que comumente leva o aluno, guiado por essa meta, a motivar-se em atividades mais simples e repetitivas (Bzuneck \& Boruchovitch, 2019; Hulleman et al., 2010; Senko \& Dawson, 2017; van Yperen, Elliot, \& Anseel, 2009).

O conjunto de diferentes estratégias de aprendizagem somada à qualidade motivacional, expressa pelo interesse, engajamento e persistência, favorecem com que os estudantes apresentem um bom desempenho escolar, aumentando as chances de conclusão do curso (Rozendaal et al., 2003; Slaats et al., 1999). O modo como o estudante percebe e lida com as demandas do curso tende a interferir na regulação do seu esforço. Nesse sentido, a motivação repercute na seleção das 
estratégias de aprendizagem que o estudante considera mais adequadas para atingir os seus objetivos de aprendizagem ou de desempenho. Por sua vez, o valor atribuído às atividades e o nível de autonomia para realiza-las são associados ao uso efetivo dessas estratégias (van Grinsven \& Tillema, 2006).

Ainda, tratando da motivação, as estratégias de aprendizagem metacognitivas se relacionam com o engajamento comportamental e emocional. Desse modo, incentivar o desenvolvimento de características motivacionais da meta aprender, juntamente com as estratégias metacognitivas, pode tornar a rotina acadêmica mais atrativa. Para tanto, se destacam as práticas pedagógicas que oportunizam a participação dos estudantes, pautadas em devolutivas construtivas e no estabelecimento de um ambiente cooperativo, capaz de fazer com que os alunos compreendam a relevância de cada tarefa para a sua formação (Bzuneck \& Sales, 2011; Rothes, Lemos, \& Gonçalves, 2017).

Em uma amostra do ensino médio técnico finlandês, Jossberger et al., (2019) observaram que os estudantes indicaram o uso de estratégias de aprendizagem profundas, nas quais se identifica uma associação entre o conhecimento prévio com os conteúdos aprendidos no curso; a utilização de estratégias de regulação interna, referentes ao planejamento, monitoramento e os ajustes na forma de estudar; e a percepção de aprendizagem como uma construção do conhecimento. Ao investigarem as associaçóes entre esses construtos, os autores verificaram que a concepçáo de aprendizagem, voltada para a repetição das informaçóes, esteve relacionada às estratégias de regulação externa e à motivação intrínseca. Ademais, as noçóes de aprendizagem como uma construção do conhecimento se relacionaram com a regulação interna e com o processamento superficial, pautado em um modo mecânico de aprender. Essa pesquisa indicou que, apesar dos estudantes apontarem para as estratégias que enfatizam a metacognição, as estratégias cognitivas, sobretudo a repetição, estão presentes na rotina dos estudantes, assim como foi averiguado no referido estudo de Scacchetti et al. (2015), com estudantes brasileiros. 
Para o presente estudo, levantou-se a hipótese que os fatores da EAVAP-EP Ausência de Estratégias Metacognitivas Disfuncionais, Estratégias Cognitivas e Estratégias Metacognitivas apresentariam correlaçóes de direção positiva com a meta aprender e negativa, com a meta performance evitaçáo. Para a meta performance aproximação, se pressupunha correlaçóes negativas com os fatores da escala que representam as estratégias de aprendizagem metacognitivas e correlação positiva com as estratégias cognitivas. Também, se presumiu que as estratégias de aprendizagem avaliadas pela EAVAP-EP seriam preditoras das metas de realização (Bahri \& Corebima, 2015; Santos \& Inácio, 2020; Scacchetti et al., 2015).

O objetivo secundário desta pesquisa foi realizar uma análise exploratória a respeito das possíveis diferenças no uso de estratégias de aprendizagem e na orientaçáo motivacional pelas metas de realização em razáo da idade dos estudantes, do período de pausa nos estudos e da quantidade de módulos cursados no ETP. Conjectura-se que essas variáveis incidem no aspecto contextual dessa modalidade de ensino e podem interferir nos tipos de estratégias de aprendizagem desenvolvidas ao longo do curso, bem como no perfil motivacional dos estudantes (Bardach et al., 2019; Bzuneck \& Sales, 2011; Rothes et al., 2017).

Ao focalizar os adultos que frequentam o ETP, se pressupóe que os estudantes sejam capazes de compreender e desenvolver estratégias mais profundas, de ordem metacognitiva (Rothes et al., 2017). Contudo, as investigaçóes indicam que os estudantes acabam utilizando as estratégias cognitivas para atenderem as demandas do curso (Jossberger et al., 2019; Scacchetti et al., 2015). Em vista disso, esta pesquisa buscou analisar a forma como os estudantes lidam com ambos os tipos de estratégias de aprendizagem.

Quanto à motivação, os estudantes adultos possuem maior facilidade para incorporar ao seu perfil motivacional as características contextuais das metas de realização transmitidas pelos seus professores. Comumente, as metas contextuais estão alinhadas com a representação de sucesso acadêmico, preconizada pela Instituição de Ensino e interferem nas suas metas pessoais de aprendizagem (Bardach et al., 2019). 


\section{Método}

\section{Participantes}

A amostra de estudo foi composta de 401 estudantes do Ensino Técnico Profissionalizante de uma escola localizada no interior do estado de São Paulo, Brasil. A idade mínima dos estudantes foi de 18 e máxima de 67 anos $(M=27.29 ; D P=9.05)$. A maior parte dos estudantes era do sexo feminino $(n=352 ; 87.8 \%)$. Os alunos frequentavam os cursos técnicos de Enfermagem $(n=164)$, Farmácia $(n=80)$, Estética $(n=56)$, Administração $(n=42)$, Turismo $(n=23)$, Recursos Humanos $(n=16)$, Moda e Logística ( $n=10$ em cada curso).

\section{Instrumentos}

Questionário de Identificação: $\mathrm{O}$ instrumento foi utilizado para coletar informaçóes relativas à idade dos participantes, sexo biológico, tipo de curso, período de pausa nos estudos e quantidade de módulos cursados.

Escala de Avaliação de Estratégias de Aprendizagem para o Ensino Profissionalizante (EAVAP-EP; Pereira \& Santos, 2016): A EAVAP-EP avalia as estratégias de aprendizagem cognitivas e metacognitivas de estudantes do Ensino Técnico Profissionalizante. Os 33 itens que a compóem são distribuídos em três fatores, a saber, Ausência de Estratégias Metacognitivas Disfuncionais, Estratégias Cognitivas e Estratégias Metacognitivas. A chave de reposta da escala é tipo Likert de três pontos em que 2 equivale à “sempre”, 1 à "às vezes” e 0 à "nunca”. Altas pontuaçôes nos seus fatores indicam a aderência às estratégias de aprendizagem avaliadas e vice-versa. A EAVAP-EP possui evidência de validade de conteúdo, evidência de validade baseada na estrutura interna e estimativas de fidedignidade (Pereira, Santos, Ferraz, \& 2020).

Escala de Avaliação da Motivação para a Aprendizagem (EMAPRE; Zenorini \& Santos, 2010): A EMAPRE avalia a motivação na perspectiva das metas de realização de estudantes do ensino básico ao ensino superior. Ela possui 28 itens que constituem três fatores que correspondem a organização das metas de realização pessoais $1 \mathrm{X} 2$ : 
Meta Aprender, Meta Performance Aproximação e Meta Performance Evitação. O formato de resposta é tipo Likert, com três pontos em que 1 corresponde à "concordo", 2 à "não sei" e 3 à "discordo". Altas pontuaçóes nos seus fatores indicam o tipo de orientação motivacional do estudante em cada uma das metas de realização. Em amostras de adultos, como é o caso da presente pesquisa, a EMAPRE possui evidência de validade baseada na estrutura interna e evidência de validade baseada na relação com outras variáveis, do tipo critério (Santos, Alcará, \& Zenorini, 2013), assim como estimativas de precisão (Dalbosco, Ferraz, \& Santos, 2018; Santos et al., 2013; Santos \& Mognon, 2016).

\section{Procedimento de coleta de dados}

O projeto obteve aprovação pelo Comitê de Ética da Instituição de Ensino Superior o qual está vinculado (No do parecer de aprovação: 971.269). A pesquisa foi conduzida com base na Resolução do 510/2016 do Conselho Nacional de Saúde (Brasil, 2016). Os estudantes consentiram participar do estudo por meio da assinatura ao Termo de Consentimento Livre e Esclarecido. Os instrumentos foram aplicados no formato lápis e papel, presencialmente e respondido pelos alunos de forma coletiva, durante o horário de aula. A coleta de dados em cada sala de aula levou, em média, 30 minutos.

\section{Análise de dados}

Softwares utilizados: Statistical Package for the Sciences (SPSS, versão 25.0); MPlus (version 7.11; Muthen \& Muthen, 2012). R (version 3.4.1; R Core Team, 2019): pacotes psych (Revelle, 2017) e GPArotation (Bernaards \& Jennrich, 2015).

Análise de correlação. Verificou-se a não normalidade dos dados da EAVAP-EP e EMAPRE por meio do teste de Shapiro Wilk, $p<.01$. Aplicou-se a correlação não paramétrica rho $(\rho)$ de Spearman. Valores de interpretação das magnitudes das correlações: $r \leq .10$, pequeno; $r \leq$ .30 , médio; $r \leq .50$, grande (Cohen, 1992).

Modelagem por equações estruturais. Estimador Maximum Likelihood Robust (MLR; Hu \& Bentler, 1999). Valores de interpretação 
do modelo. Razão do $\chi^{2} / g l>.05$; Root Mean Square Error of Approximation, IC 90\%, RMSEA <.05 à .10; Comparative Fit Index (CFI) e Tucker-Lewis Index (TLI) < .80 e > .95 (Marôco, 2014).

Análise de quartis. Formação de grupos das VIs idade, pausa nos estudos e quantidade de módulos cursados.

Análise Multivariada da Variância (MANOVA), tendo como VDs os três fatores da EAVAP-EP e VIs a idade, a pausa nos estudos e a quantidade de módulos cursados. Os dados atenderam três critérios para a execução da MANOVA (2, 3 e 4): (1) O pressuposto de normalidade não foi totalmente atendido (Shapiro Wilk, $p<.01$ ). (2) Os dados apresentaram homogeneidade de variâncias-covariâncias $(p=.39)$. (3) Os dados não apresentaram outliers multivariados (distância máxima de Mahalanobis para três VDs = 13.90). (4) Verificou-se a ausência de multicolinearidade, $r<.90$ entre as VDs. (5) Os dados não apresentaram relação linear.

Análise Multivariada da Variância (MANOVA), tendo como VDs os três fatores da EMAPRE e VIs a idade, a pausa nos estudos e a quantidade de módulos cursados. Foi preenchido um critério para a realização da MANOVA (4): (1) A premissa de normalidade não atendida (Shapiro Wilk, $p<.01)$. (2) Os dados não apresentaram homogeneidade de variâncias-covariâncias $(p<.001)$. (3) Os dados apresentaram outliers multivariados (distância máxima de Mahalanobis para três VDs = 30.87). (4) Verificou-se a ausência de multicolinearidade, $r<.90$ entre as VDs. (5) Os dados não apresentaram relação linear.

Para a visualização das diferenças de média nas VDs em razão das VIs solicitou-se o teste post-hoc de Tukey. Em decorrência da violação desses critérios, considerou-se a significância estatística dos resultados das MANOVAs por meio do teste Traço de Pillai, $p<.05$.

Averiguou-se o tamanho do efeito dos resultados das comparaçóes pela MANOVA por meio do eta ao quadrado parcial $\left(\eta \mathrm{p}^{2}\right)$. Valores de interpretação: $\eta p^{2}<0,01$, trivial; entre .01 e .05 , pequeno; entre .06 e .13 , médio; $\eta p^{2}>.14$, grande (Jasp, 2018).

As estatísticas descritivas (médias e desvios-padrão), apresentados na Tabela 1 e nos resultados dos testes de post-hoc de Tukey, repre- 
sentam a média ponderada dos valores das chaves de resposta dos testes. EAVAP-EP: 0 a 2; EMAPRE-EP: 1 a 3.

Estimativas de fidedignidade foram avaliadas por meio do coeficiente alfa $(\alpha)$ e ômega de McDonald's ( $\omega$ total). Valores de interpretação de ambos os índices de confiabilidade: $\alpha$ e $\omega>.70$ são classificados como adequados (Dunn et al., 2013).

\section{Resultados}

O resultado das análises descritivas da EAVAP-EP, exposto na Tabela 1, mostra que os estudantes tenderam a assinalar mais o fator Estratégias Metacognitivas e menos o fator Estratégias Cognitivas. $\mathrm{Na}$ EMAPRE, os alunos pontuaram mais no fator Meta Aprender e menos no fator Meta Performance Evitação. Com exceção do coeficiente $\alpha<.70$ no fator da EAVAP-EP Estratégias Metacognitivas, os demais fatores obtiveram valores de confiabilidade adequados.

Para investigar a evidência de validade baseada na relação com outras variáveis, para a EAVAP-EP com a EMAPRE, examinou-se as correlaçóes existentes entre os fatores de ambos os instrumentos. Pelos dados da Tabela 1 nota-se que o fator da EAVAP-EP Ausência de Estratégias Metacognitivas Disfuncionais apresentou uma correlação de direção positiva com o fator da EMAPRE Meta Aprender, indicativo de que as estratégias metacognitivas, quando funcionais, se associam às características desse perfil motivacional. Isso é corroborado pela intensidade média identificada na associação de ambos os construtos. Correlações de direção negativa e de pequena magnitude foram identificadas entre o fator Ausência de Estratégias Metacognitivas Disfuncionais e os fatores Meta Performance Aproximação e Meta Performance Evitação. A pequena intensidade das correlaçóes aponta para a menor percepção na ausência de estratégias metacognitivas disfuncionais em alunos orientados pelas metas performances. Acrescenta-se que o sentido negativo das correlaçóes sinaliza que a presença de estratégias metacognitivas, que caracteriza os estudantes com altas pontuaçóes nesse fator da EAVAP-EP, diverge de perfis motivacionais que priorizam o desempenho no ensino profissionalizante. 
No fator Estratégias Cognitivas da EAVAP-EP a correlação positiva e de grande magnitude indicam que os estudantes do EP, que possuem características da meta aprender, se utilizam desse tipo de estratégias na sua rotina de estudos. Em oposição, a correlação desse fator da EAVAP-EP com o fator da EMAPRE Meta Performance Evitação foi negativa e de pequena magnitude, sugerindo que a busca por informaçóes, viabilizada pelas estratégias cognitivas, não corresponde as açōes dos estudantes com esse perfil motivacional.

Para o fator da EAVAP-EP Estratégias Metacognitivas identificou-se significância estatística somente para a Meta Aprender da EMAPRE. O sentido positivo dessa correlação é associado à capacidade dos alunos guiados pela meta aprender a regularem os aspectos cognitivos para atender as demandas acadêmica. Todavia, a magnitude dessa relação no ensino profissionalizante foi pequena.

\section{Tabela 1}

Correlaçôes rho de Spearman entre a EAVAP-EP e a EMAPRE

\begin{tabular}{ccccccccc}
\hline Fatores & $M$ & $D P$ & EAVAP1 & EAVAP2 & EAVAP3 & EMAPRE1 & EMAPRE2 & EMAPRE3 \\
\hline EAVAP1 & 1.23 & .31 & $\begin{array}{c}\alpha=.78 \\
\omega=.82\end{array}$ & & & & \\
EAVAP2 & 1.13 & .36 & $.37^{* *}$ & $\begin{array}{c}\alpha=.84 \\
\omega=.85\end{array}$ & & & & \\
EAVAP3 & 1.54 & .33 & -.2 & .08 & $\alpha=.61$ & & \\
& & & & & $\omega=.72$ & & \\
EMAPRE1 & 2.67 & .32 & $.47^{* *}$ & $.51^{* *}$ & $.16^{*}$ & $\alpha=.78$ & & \\
& & & & & & $\omega=.82$ & & \\
EMAPRE2 & 1.58 & .49 & $-.15^{*}$ & -.05 & -.06 & -.04 & $\alpha=.82$ & \\
& & & & & & & & \\
EMAPRE3 & 1.34 & .42 & $-.28^{* *}$ & $-.14^{*}$ & -.08 & $-.22^{* *}$ & $.14^{*}$ & $\alpha=.79$ \\
& & & & & & & & $\omega=.85$ \\
\hline
\end{tabular}

Legenda. EAVAP1 = Ausência de Estratégias Metacognitivas Disfuncionais; EAVAP2 = Estratégias Cognitivas; EAVAP3 = Estratégias Metacognitivas; EMAPRE1 = Meta Aprender; EMAPRE2 = Meta Performance Aproximação; EMAPRE3 = Meta Performance Evitação; $\alpha=$ coeficiente alfa; $\omega$ = ômega total.

$$
{ }^{*} p<.01 ;{ }^{* *} p<.001 .
$$


Para alcançar a evidência de validade baseada na relação com outras variáveis, analisou-se, na sequência, o potencial explicativo da EAVAP-EP para os fatores da EMAPRE. O modelo obtido pela modelagem por equaçóes estruturais (Figura 1 ) apresentou $\chi^{2}=2498.009 ; g l$ $=1754(p<.001)$, sendo a razão de $\chi^{2} / g l=1.42$. Os índices de ajuste são classificados como muito bons para o RMSEA = .03 (90\% IC .03 - .04); e bom para o CFI = .94 e TLI = .93.

Conforme ilustra a Figura 1, a EAVAP-EP explica a variância dos três fatores da EMAPRE, sendo 41\% da Meta Performance Aproximação, $40 \%$ da Meta Aprender e $12 \%$ da Meta Performance Evitação. Apoiado nesse resultado, é previsto que as estratégias de aprendizagem cognitivas e metacognitivas sejam predominantes nas orientaçóes motivacionais, cujo foco é o de evidenciar o bom desempenho no curso e adquirir conhecimento a partir da formaçáo profissionalizante. Também, é previsto que elas estejam pouco presentes nas açóes de estudantes, orientados pelo receio de serem expostos pelo baixo desempenho.

O valor dos coeficientes de regressão, bem como a sua direção, negativa ou positiva, sinalizam que a contribuição das estratégias de aprendizagem avaliadas EAVAP-EP é distinta em cada uma das metas de realização. Conforme exposto na Figura 1, os Fatores da EAVAP-EP Estratégias Metacognitivas Disfuncionais e Estratégias Cognitivas tiveram os $\beta$ negativos para a Meta Performance Aproximação, indicando que o uso dessas estratégias pode interferir na diminuição de certos atributos desse perfil motivacional, com destaque para as estratégias metacognitivas. A mesma linha de raciocínio é aplicada a ambos os fatores da EAVAP-EP e à meta performance evitação, com o diferencial de as estratégias cognitivas apresentarem um $\beta$ maior para esse tipo de orientação motivacional. Todos os fatores da EAVAP-EP tiveram $\beta$ positivos, indicando que o conjunto dos diversos tipos de estratégias de aprendizagem tem o potencial de sustentar a motivaçáo dos estudantes do ensino profissionalizante pela meta aprender. 


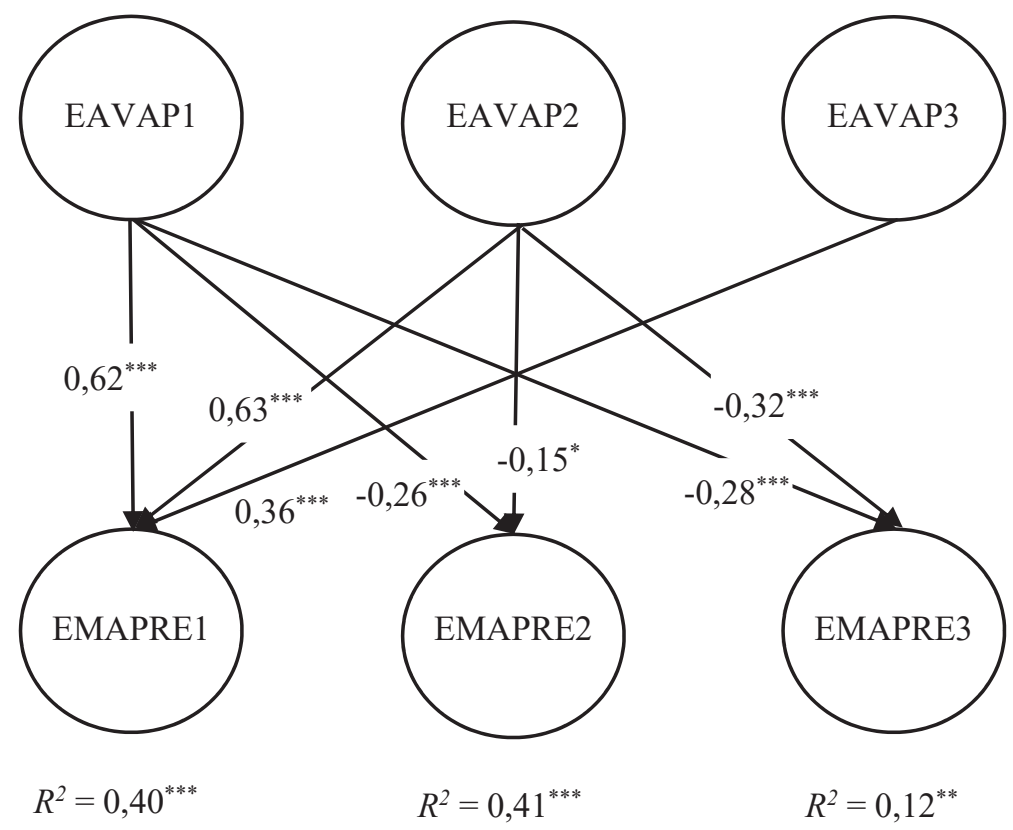

Figura 1. Modelagem por Equaçóes Estruturais - Fatores da EAVAP-EP (Variáveis Independentes); Fatores da EMAPRE (Variáveis Dependentes) Legenda. EAVAP1 = Ausência de Estratégias Metacognitivas Disfuncionais; EAVAP2 = Estratégias Cognitivas; EAVAP3 = Estratégias Metacognitivas; EMAPRE1 = Meta Aprender; EMAPRE2 = Meta Performance Aproximação; EMAPRE3 = Meta Performance Evitação ${ }^{*} p<.05 ;{ }^{* *} p<.01 ;{ }^{* * *} p<.001$ 
Este estudo prossegue com o intento de atender o seu objetivo secundário, centralizado na investigaçáo de possíveis diferenças no funcionamento das estratégias de aprendizagem e das metas de realização dos alunos do EP, quanto à idade, o período de pausa nos estudos e à quantidade de módulos cursados. As três VIs foram divididas em três grupos por meio da análise de quartis. Idade: $\mathrm{G}_{\text {idadel }}$, estudantes com 18 a 19 anos; $G_{\text {idade2 }}$, 20 a 32 anos; $G_{\text {idade3 }}$, acima de 33 anos. Período de pausa nos estudos: $G_{\text {pausal }}$, pausa de até um ano; $G_{\text {pausa2' }}$, pausa de um ano e um mês até nove anos e onze meses; $G_{\text {pausa3 }}$, pausa de dez anos ou mais. Quantidade de módulos cursados: $G_{\text {mód1 }}$ formado por alunos que tinham cursado até três módulos do curso; o $\mathrm{G}_{\text {mód2 }}$, de quatro a seis módulos; e o $\mathrm{G}_{\text {mód3 }}$, com sete ou mais módulos.

Os resultados da MANOVA, reportados na Tabela 2, mostram que para a amostra investigada, a idade foi a única variável que demonstrou ter efeito para as estratégias cognitivas, bem como a ausência de estratégias metacognitivas disfuncionais na rotina acadêmica no ETP.

Verificou-se que os estudantes com maior idade $\left(\mathrm{G}_{\text {idades }}\right)$ indicaram mais a ausência de estratégias metacognitivas disfuncionais, em comparação aos estudantes mais jovens ( $\mathrm{G}_{\text {idadel }}$ e $\mathrm{G}_{\text {idade? }}$, respectivamente). Os estudantes do $G_{\text {idade3 }}$ também se destacaram no uso das estratégias cognitivas, quando comparados com o $\mathrm{G}_{\text {idade } 2}$ e $\mathrm{G}_{\text {idadel. }}$ Ainda considerando a variável idade, a prova post-hoc de Tukey não indicou diferenciação de média para as estratégias metacognitivas. Para as variáveis pausa nos estudos e quantidade de módulos cursados, náo foram verificadas diferenças estatisticamente significativas na utilização das estratégias de aprendizagem. 
Relações entre as estratégias de aprendizagem e motivação no Ensino Técnico / Ferraz et al.

\section{Tabela 2}

Análise de Variância MANOVA: Diferenciaçóes das Estratégias de Aprendizagem Quanto à Idade, Pausa nos Estudos e Módulos Cursados

\begin{tabular}{|c|c|c|c|c|c|c|}
\hline \multirow[t]{2}{*}{ VD } & \multirow[t]{2}{*}{$F^{*}$} & \multirow[t]{2}{*}{ Idade } & \multirow[t]{2}{*}{$N$} & \multirow[t]{2}{*}{ Fatores da EAVAP-EP } & \multicolumn{2}{|c|}{$\begin{array}{l}\text { Subconjuntos } \\
\text { post-hoc de Tukey }\end{array}$} \\
\hline & & & & & 1 & 2 \\
\hline \multirow{12}{*}{$\begin{array}{l}\text { Estratégias de } \\
\text { aprendizagem } \\
\text { (EAVAP-EP) }\end{array}$} & \multirow{12}{*}{$\begin{array}{c}4.770 \\
p<.001 \\
\eta p^{2}=.042\end{array}$} & $\mathrm{G}_{\text {idade1 }}$ & 99 & \multirow{4}{*}{$\begin{array}{l}\text { Ausência de Estratégias } \\
\text { Metacognitivas Disfuncionais }\end{array}$} & 1.18 & \\
\hline & & $\mathrm{G}_{\text {idade2 }}$ & 157 & & 1.21 & \\
\hline & & $\mathrm{G}_{\text {idade3 }}$ & 93 & & & 1.32 \\
\hline & & $P$ & & & .754 & 1.000 \\
\hline & & $\mathrm{G}_{\text {idade1 }}$ & 99 & \multirow[t]{4}{*}{ Estratégias Cognitivas } & 1.13 & \\
\hline & & $\mathrm{G}_{\text {idade2 }}$ & 157 & & 1.10 & \\
\hline & & $\mathrm{G}_{\text {idade3 }}$ & 93 & & & 1.26 \\
\hline & & $P$ & & & .706 & 1.000 \\
\hline & & $\mathrm{G}_{\text {idade1 }}$ & 99 & \multirow[t]{4}{*}{ Estratégias Metacognitivas } & 1.59 & \\
\hline & & $\mathrm{G}_{\text {idade2 }}$ & 157 & & 1.50 & \\
\hline & & $\mathrm{G}_{\text {idade3 }}$ & 93 & & 1.58 & \\
\hline & & $P$ & & & .143 & \\
\hline \multirow[t]{2}{*}{ VD } & \multirow[t]{2}{*}{$F$} & \multirow{2}{*}{$\begin{array}{c}\text { Pausa } \\
\text { nos } \\
\text { estudos }\end{array}$} & \multirow[t]{2}{*}{$N$} & \multirow[t]{2}{*}{ Fatores da EAVAP-EP } & \multicolumn{2}{|c|}{$\begin{array}{c}\text { Subconjuntos } \\
\text { post-hoc de Tukey }\end{array}$} \\
\hline & & & & & 1 & \\
\hline
\end{tabular}




\begin{tabular}{|c|c|c|c|c|c|c|}
\hline \multirow[t]{2}{*}{ VD } & \multirow[t]{2}{*}{$F^{*}$} & \multirow[t]{2}{*}{ Idade } & \multirow[t]{2}{*}{$N$} & \multirow[t]{2}{*}{ Fatores da EAVAP-EP } & \multicolumn{2}{|c|}{$\begin{array}{c}\text { Subconjuntos } \\
\text { post-hoc de Tukey }\end{array}$} \\
\hline & & & & & 1 & 2 \\
\hline \multirow{12}{*}{$\begin{array}{l}\text { Estratégias de } \\
\text { aprendizagem } \\
\text { (EAVAP-EP) }\end{array}$} & \multirow{12}{*}{$\begin{array}{c}3.448 \\
p<.01 \\
\eta p^{2}=.031\end{array}$} & $\mathrm{G}_{\text {pausa1 }}$ & 146 & \multirow{4}{*}{$\begin{array}{l}\text { Ausência de Estratégias } \\
\text { Metacognitivas Disfuncionais }\end{array}$} & 1.23 & \\
\hline & & $\mathrm{G}_{\text {pausa2 }}$ & 72 & & 1.24 & \\
\hline & & $\mathrm{G}_{\text {pausa3 }}$ & 131 & & 1.24 & \\
\hline & & $P$ & & & .951 & \\
\hline & & $\mathrm{G}_{\text {pausa } 1}$ & 146 & \multirow[t]{4}{*}{ Estratégias Cognitivas } & 1.18 & \\
\hline & & $\mathrm{G}_{\text {pausa2 }}$ & 72 & & 1.13 & \\
\hline & & $\mathrm{G}_{\text {pausa3 }}$ & 131 & & 1.12 & \\
\hline & & $P$ & & & .400 & \\
\hline & & $\mathrm{G}_{\text {pausa } 1}$ & 146 & \multirow[t]{4}{*}{ Estratégias Metacognitivas } & 1.51 & \\
\hline & & $\mathrm{G}_{\text {pausa } 2}$ & 72 & & 1.53 & \\
\hline & & $\mathrm{G}_{\text {pausa3 }}$ & 131 & & 1.59 & \\
\hline & & $P$ & & & .194 & \\
\hline \multirow[t]{2}{*}{ VD } & \multirow[t]{2}{*}{$F$} & \multirow[t]{2}{*}{$\begin{array}{l}\text { Módulos } \\
\text { cursados }\end{array}$} & \multirow[t]{2}{*}{$N$} & \multirow[t]{2}{*}{ Fatores da EAVAP-EP } & \multicolumn{2}{|c|}{$\begin{array}{c}\text { Subconjuntos } \\
\text { post-hoc de Tukey }\end{array}$} \\
\hline & & & & & 1 & 2 \\
\hline \multirow{12}{*}{$\begin{array}{l}\text { Estratégias de } \\
\text { aprendizagem } \\
\text { (EAVAP-EP) }\end{array}$} & \multirow{12}{*}{$\begin{array}{c}.307 \\
p=.9 \\
\eta p^{2}=.003\end{array}$} & $\mathrm{G}_{\text {mód1 }}$ & 106 & \multirow{4}{*}{$\begin{array}{c}\text { Ausência de Estratégias } \\
\text { Metacognitivas Disfuncionais }\end{array}$} & 1.27 & \\
\hline & & $\mathrm{G}_{\text {mód2 }}$ & 151 & & 1.19 & \\
\hline & & $\mathrm{G}_{\text {mód3 }}$ & 92 & & 1.26 & \\
\hline & & $P$ & & & .090 & \\
\hline & & $\mathrm{G}_{\text {mód1 }}$ & 106 & \multirow[t]{4}{*}{ Estratégias Cognitivas } & 1.12 & \\
\hline & & $\mathrm{G}_{\text {mód2 }}$ & 151 & & 1.11 & \\
\hline & & $\mathrm{G}_{\text {mód3 } 3}$ & 92 & & & 1.25 \\
\hline & & $P$ & & & 1.000 & 1.000 \\
\hline & & $\mathrm{G}_{\text {mód1 }}$ & 106 & \multirow[t]{4}{*}{ Estratégias Metacognitivas } & 1.56 & \\
\hline & & $\mathrm{G}_{\text {mód2 }}$ & 151 & & 1.53 & \\
\hline & & $\mathrm{G}_{\text {mód3 }}$ & 92 & & 1.57 & \\
\hline & & $P$ & & & .592 & \\
\hline
\end{tabular}

Nota. *significância estatística com base no teste Traço de Pillai; $g l=6$. 
Ao focalizar a motivação dos estudantes para lidar com o cotidiano do EP, identificou-se a existência de efeito da idade e do período de pausa nos estudos para as metas de realizaçáo (Tabela 3). $\mathrm{O}$ mesmo não ocorreu para a variável quantidade de módulos cursados.

A prova post-hoc de Tukey indicou haver distinção de médias apenas para o perfil motivacional da meta performance aproximação. Os estudantes de maior faixa etária eram menos orientados por essa meta do que os alunos mais jovens $\left(\mathrm{G}_{\text {idade1 }}\right.$ e $\mathrm{G}_{\text {idade22 }}$, respectivamente). $\mathrm{Na}$ variável pausa de estudos, também se averiguou diferenciação de médias na meta performance aproximação. Os estudantes que haviam pausado o período intermediário $\left(\mathrm{G}_{\text {pausa2 }}\right)$ tinham mais características dessa meta de realização em comparação aos alunos que fizeram uma pausa de até um ano $\left(\mathrm{G}_{\text {pausal }}\right)$.

\section{Tabela 3}

Análise de Variância MANOVA: Diferenciações das Metas de Realização Quanto à Idade, Pausa nos Estudos e Módulos Cursados

\begin{tabular}{|c|c|c|c|c|c|c|}
\hline \multirow[t]{2}{*}{ VD } & \multirow[t]{2}{*}{$F^{*}$} & \multirow[t]{2}{*}{ Idade } & \multirow[t]{2}{*}{$N$} & \multirow[t]{2}{*}{ Fatores da EAVAP-EP } & \multicolumn{2}{|c|}{$\begin{array}{c}\text { Subconjuntos } \\
\text { post-hoc de Tukey }\end{array}$} \\
\hline & & & & & 1 & 2 \\
\hline \multirow{12}{*}{$\begin{array}{c}\text { Metas de } \\
\text { realizaçáo } \\
\text { (EMAPRE) }\end{array}$} & \multirow{12}{*}{$\begin{array}{c}2.531 \\
p=.02 \\
\eta p^{2}=.023\end{array}$} & $\mathrm{G}_{\text {idade1 }}$ & 98 & \multirow[t]{4}{*}{ Meta Aprender } & 2.65 & \\
\hline & & $\mathrm{G}_{\text {idade2 }}$ & 153 & & 2.66 & \\
\hline & & $\mathrm{G}_{\text {idade3 }}$ & 92 & & 2.73 & \\
\hline & & $P$ & & & .124 & \\
\hline & & $\mathrm{G}_{\text {idadel }}$ & 98 & \multirow{4}{*}{$\begin{array}{c}\text { Meta Performance } \\
\text { Aproximação }\end{array}$} & & 1.71 \\
\hline & & $\mathrm{G}_{\text {idade2 }}$ & 153 & & \multirow{3}{*}{1.41} & 1.59 \\
\hline & & $\mathrm{G}_{\text {idade3 }}$ & 92 & & & \\
\hline & & $P$ & & & & \\
\hline & & $\mathrm{G}_{\text {idade1 }}$ & 98 & \multirow[t]{4}{*}{ Meta Performance Evitação } & 1.41 & \\
\hline & & $\mathrm{G}_{\text {idade2 }}$ & 153 & & 1.34 & \\
\hline & & $\mathrm{G}_{\text {idade3 }}$ & 92 & & 1.27 & \\
\hline & & $P$ & & & .051 & \\
\hline
\end{tabular}




\begin{tabular}{|c|c|c|c|c|c|c|}
\hline \multirow[t]{2}{*}{ VD } & \multirow[t]{2}{*}{$F$} & \multirow{2}{*}{$\begin{array}{c}\text { Pausa } \\
\text { nos } \\
\text { estudos }\end{array}$} & \multirow[t]{2}{*}{$N$} & \multirow[t]{2}{*}{ Fatores da EMAPRE } & \multicolumn{2}{|c|}{$\begin{array}{c}\text { Subconjuntos } \\
\text { post-hoc de Tukey }\end{array}$} \\
\hline & & & & & 1 & 2 \\
\hline \multirow{12}{*}{$\begin{array}{l}\text { Metas de } \\
\text { realização } \\
\text { (EMAPRE) }\end{array}$} & \multirow{12}{*}{$\begin{array}{c}1.229 \\
p=.2 \\
\eta p^{2}=.012\end{array}$} & $\mathrm{G}_{\text {pausa1 }}$ & 70 & \multirow[t]{4}{*}{ Meta Aprender } & 2.64 & \\
\hline & & $\mathrm{G}_{\text {pausa2 }}$ & 145 & & 2.67 & \\
\hline & & $\mathrm{G}_{\text {pausa3 }}$ & 128 & & 2.70 & \\
\hline & & $P$ & & & & \\
\hline & & $\mathrm{G}_{\text {pausa } 1}$ & 70 & \multirow{4}{*}{$\begin{array}{c}\text { Meta Performance } \\
\text { Aproximação }\end{array}$} & \multirow[t]{2}{*}{1.40} & \\
\hline & & $\mathrm{G}_{\text {pausa2 }}$ & 145 & & & 1.58 \\
\hline & & $\mathrm{G}_{\text {pausa3 }}$ & 128 & & 1.56 & 1.56 \\
\hline & & $P$ & & & .050 & .129 \\
\hline & & $\mathrm{G}_{\text {pausa } 1}$ & 70 & \multirow[t]{4}{*}{ Meta Performance Evitação } & 1.35 & \\
\hline & & $\mathrm{G}_{\text {pausa2 }}$ & 145 & & 1.34 & \\
\hline & & $\mathrm{G}_{\text {pausa3 }}$ & 128 & & 1.33 & \\
\hline & & $P$ & & & .953 & \\
\hline \multirow[t]{2}{*}{ VD } & \multirow[t]{2}{*}{$F^{*}$} & \multirow[t]{2}{*}{$\begin{array}{l}\text { Módulos } \\
\text { cursados }\end{array}$} & \multirow[t]{2}{*}{$N$} & \multirow[t]{2}{*}{ Fatores da EMAPRE } & \multicolumn{2}{|c|}{$\begin{array}{c}\text { Subconjuntos } \\
\text { post-hoc de Tukey }\end{array}$} \\
\hline & & & & & 1 & 2 \\
\hline \multirow{12}{*}{$\begin{array}{l}\text { Metas de } \\
\text { realização } \\
\text { (EMAPRE) }\end{array}$} & \multirow{12}{*}{$\begin{array}{c}1.907 \\
p=.8 \\
\eta p^{2}=.018\end{array}$} & $\mathrm{G}_{\text {mód1 }}$ & 104 & \multirow[t]{4}{*}{ Meta Aprender } & 2.65 & \\
\hline & & $\mathrm{G}_{\text {mód2 }}$ & 147 & & 2.65 & \\
\hline & & $\mathrm{G}_{\text {mód3 } 3}$ & 92 & & 2.71 & \\
\hline & & $P$ & & & .402 & \\
\hline & & $\mathrm{G}_{\text {mód1 }}$ & 104 & \multirow{4}{*}{$\begin{array}{c}\text { Meta Performance } \\
\text { Aproximação }\end{array}$} & 1.51 & \\
\hline & & $\mathrm{G}_{\text {mód2 }}$ & 147 & & 1.63 & \\
\hline & & $\mathrm{G}_{\text {mód3 } 3}$ & 92 & & 1.56 & \\
\hline & & $P$ & & & & \\
\hline & & $\mathrm{G}_{\text {mód1 }}$ & 104 & \multirow[t]{4}{*}{ Meta Performance Evitação } & 1.29 & \\
\hline & & $\mathrm{G}_{\text {mód2 }}$ & 147 & & 1.39 & \\
\hline & & $\mathrm{G}_{\text {mód3 }}$ & 92 & & 1.32 & \\
\hline & & $P$ & & & & \\
\hline
\end{tabular}

Nota. *significância estatística com base no teste Traço de Pillai; $g l=6$. 


\section{Discussão}

Mediante a conferência da evidência de validade, baseada na relação com outras variáveis da EAVAP-EP com a EMAPRE, alcançou-se o objetivo principal deste estudo. $\mathrm{O}$ uso de estratégias cognitivas esteve correlacionado com as características da meta aprender, com destaque para a manutenção da motivação manifesta pela persistência e pelo interesse do aluno do Ensino Técnico Profissionalizante nos estudos (Santos \& Inácio, 2020; Scacchetti et al., 2015). O fator da EAVAP-EP Ausência de Estratégias Metacognitivas Disfuncionais, quando comparado com o fator Estratégias Metacognitivas, apresentou um valor de correlação com a meta aprender de magnitude mais alta, assim como um nível maior de significância. Isso sugere que no ETP, a percepção dos estudantes sobre a ausência de estratégias cognitivas disfuncionais está mais relacionada com a sua motivação para aprendizagem do que para a utilizaçáo das estratégias metacognitivas. Todavia, é preciso ponderar que as estimativas de fidedignidade do fator Estratégias Metacognitivas ficaram aquém do adequado para a amostra avaliada. Isso também fora observado em outras versóes da EAVAP-EP, voltadas para o Ensino Técnico Profissional (Scacchetti et al., 2015) e para o Ensino Básico (Santos \& Inácio, 2020).

A correlação negativa do fator Ausência de Estratégias Metacognitivas Disfuncionais com as metas performances são consoantes ao que fora verificado no estudo de Santos e Inácio (2020). A magnitude pequena dessas correlações pode ser um indicativo de que a dificuldade de perceber que o uso de estratégias metacognitivas disfuncionais é um ponto a ser considerado ao lidar com estudantes orientados pelas metas performances, uma vez que eles concentram os seus esforços para o desempenho, podendo não refletir com maior profundidade sobre o uso inadequado das estratégias de aprendizagem do tipo metacognitivo (Bzuneck \& Boruchovitch, 2019; Senko \& Dawson, 2017; van Grinsven \& Tillema, 2006; van Yperen et al., 2009).

Ressalta-se, também, a possível falta de domínio dos estudantes orientados pela meta performance evitação em utilizar as estratégias 
cognitivas. É provável que as dificuldades dos alunos guiados por esse tipo de perfil motivacional não se limitem às estratégias metacognitivas, mas também em procedimentos de estudo considerados mais superficiais, como é o caso de reler, resumir e aplicar o conteúdo aprendido (Santos \& Inácio, 2020; Scacchetti et al., 2015). À vista disso, além de se atentar para prejuízos na qualidade da aprendizagem na perspectiva da meta performance evitação, é preciso considerar os impactos negativos que incidem sobre as expectativas dos estudantes para concluir o curso, uma vez que a desmotivaçáo e a desistência são observadas nesse perfil motivacional (Bzuneck \& Boruchovitch, 2019; van Yperen et al., 2009).

A identificação da evidência de validade baseada nas relaçóes com outras variáveis para a EAVAP foi endossada com a confirmação da sua capacidade de predizer as características motivacionais dos estudantes, o que vai ao encontro com pesquisas anteriores, que forneceram indícios desse tipo de associação (Jossberger et al., 2019; Santos \& Inácio, 2020; Slaats et al., 1999; van Grinsven, \& Tillema, 2006). A contribuição das estratégias de aprendizagem varia de acordo com o tipo de meta de realização. Essa variação é discutida sob a lógica de que a orientação por essas metas não é algo estático, ou seja, elas podem ser alteradas por meio de motivos pessoais e de razóes contextuais, identificadas pelo aluno no ambiente de ensino (Bzuneck \& Boruchovitch, 2019; Jossberger et al., 2019).

A partir dos resultados que focalizam a predição dos fatores da EAVAP-EP para as metas de realização, conjectura-se por um lado, que a capacidade de perceber a ausência ou a presença de estratégias metacognitivas disfuncionais, assim como a indicaçáo do uso desse tipo de estratégia de aprendizagem envolve um nível mais elevado de consciência dos estudantes sobre as suas açóes e isso prediz a orientação pela meta aprender (Bardach et al., 2019; Bzuneck \& Boruchovitch, 2019). Por outro lado, a direção negativa dos coeficientes betas do fator Ausência de Estratégias Metacognitivas Disfuncionais para as metas performances, é um indicativo de que introduzir os conceitos sobre as estratégias metacognitivas, em alunos guiados por essas metas, pode 
alterar o seu sistema de crenças que enfatiza o desempenho e o mérito acadêmico. Pressupóe-se que esse tipo de prática pedagógica tende a ser benéfica principalmente na meta performance evitação, na qual se observa um grande sofrimento psicológico em decorrência do receio do estudante de ser exposto por sua incapacidade autopercebida (Bzuneck \& Boruchovitch, 2019; Hulleman et al., 2010; van Yperen et al., 2009).

Nesse seguimento, aponta-se para a contribuição preditiva das estratégias cognitivas para a meta aprender, assim como se destaca que os procedimentos empregados nesse tipo de estratégia de aprendizagem podem minimizar os impactos negativos resultantes da ênfase das metas performances aproximação e evitação para o desempenho. Essa suposição é feita com base nos valores do coeficiente beta e, novamente, sinaliza que o estímulo às estratégias de aprendizagem cognitivas apoiaria, principalmente, os estudantes orientados pela meta performance evitação. Em princípio pode ser mais fácil de desenvolver esse tipo de estratégia, devido a sua compatibilidade com as especificidades do currículo do ETP (Jossberger et al., 2019; Rothes et al., 2017; Scacchetti et al., 2015; Slaats et al., 1999).

Avançando nos objetivos desta pesquisa, cabe discutir os resultados da investigação exploratória em que foi verificado que os estudantes acima de 33 anos se diferenciaram dos alunos de menor faixa etária nos fatores da EAVAP-EP Ausência de Estratégias Metacognitivas Disfuncionais e Estratégias Cognitivas. Rothes et al (2017) consideram que os estudantes com mais idade podem apresentar maior acurácia ao apontarem para aquilo que não costumam fazer, o que pode ser aplicado à indicação de ambos os fatores da EAVAP-EP. Outro ponto a se destacar é que existe uma consonância na indicação desses estudantes para as estratégias cognitivas que, supostamente, acabam sendo mais reportadas no ETP em comparação às estratégias metacognitivas (Jossberger et al., 2019; Scacchetti et al., 2015).

Ao tratar da motivação, os estudantes que tinham até 32 anos apresentaram mais características motivacionais da meta performance aproximação do que os estudantes acima de 33 anos. Estudos anteriores 
com esse público não analisaram as diferenciações da idade na motivação dos estudantes do ETP (ex. Jossberger et al., 2019; Rothes et al., 2017; Rozendaal et al., 2003; van Beek et al., 2014; van Grinsven, \& Tillema, 2006). Esse tipo de comparação é mais recorrente em outros níveis de ensino em que se compara, por exemplo, as distinçóes motivacionais em decorrência da idade em um mesmo nível de ensino na educação básica (Almeida \& Guisande, 2010). As pesquisas também apontam para a investigação de diferenças nas metas de realização nos níveis de ensino básico e superior (Bardach et al., 2019), o que não inclui a inserção do ETP. Outra variável que apresentou diferenciação nas metas de realização foi o tempo de pausa dos estudos. Estudantes que fizeram uma pausa, classificada de intermediária de um a nove anos e onze meses, demonstraram maior aderência para a meta performance aproximação do que aqueles que tiveram um período menor de pausa.

Uma vez que as estratégias de aprendizagem e a motivação se diferenciaram em razão da idade e, conforme também fora averiguado neste estudo, os tipos de estratégias estão associados com as metas de realização, cabe analisar em pesquisas futuras, o papel dessa variável para a forma como o estudante lida com a sua rotina de estudos no ETP. Por se tratar de um público adulto, que apresenta uma ampla faixa etária, recomenda-se que essa variável seja abordada em termos das vivências dos estudantes quanto às suas experiências escolares anteriores (ex. no nível básico de ensino), às suas expectativas e satisfação com o curso profissionalizante, bem como forneça detalhes sobre a sua situação trabalhista (Rothes et al., 2017). Essa indicação também se sustenta na identificação do efeito da pausa nos estudos para a aderência dos estudantes à meta performance aproximaçáo, que guia o estudante a se dedicar em tarefas que denotam maiores chances de obter reconhecimento pelo seu mérito, não se preocupando intrinsecamente com a qualidade da formação recebida (Bzuneck \& Boruchovitch, 2019; Hulleman et al., 2010; Scacchetti et al., 2014).

Apoiado nos resultados desta pesquisa sugere-se o uso da EAVAP-EP para a condução de futuras pesquisas com estudantes do ETP. Cabe analisar os índices de confiabilidade da escala, principalmente 
no fator Estratégias Metacognitivas, em outras amostras desse tipo de ensino, como uma forma de monitorar de que maneira ele se comporta em outros ambientes de testagem.

Visto que a EAVAP-EP apresentou evidência de validade baseada na relação com outras variáveis e possui evidências de validade de conteúdo e baseada na estrutura interna, bem como estimativas de fidedignidade (Pereira et al,, 2020), aventa-se também que a escala seja utilizada por coordenadores pedagógicos e professores do ETP para avaliar em que medida os estudantes conhecem e utilizam as estratégias de aprendizagem. Pressupóe-se que a EAVAP-EP sirva para auxiliar esses profissionais nas práticas pedagógicas, mais especificamente no desenvolvimento das estratégias de aprendizagem cognitivas e metacognitivas.

Conjectura-se, ainda, que o desenvolvimento das estratégias de aprendizagem também tem o potencial de interferir na motivação dos estudantes, a partir do fortalecimento de crenças mais funcionais em relação à aprendizagem previstas na meta aprender e que, em partes, também está presente na meta performance aproximação, dado que o aumento do conhecimento intelectual pode estar alinhado a bons níveis de desempenho no curso, sem que a qualidade da aprendizagem seja comprometida. Com isso, é possível fortalecer o bem-estar psicológico dos estudantes, comumente comprometido pela meta performance evitação, posto que o uso adequado das estratégias de aprendizagem pode se configurar em um fator protetivo para evitar a desmotivação. Por fim, espera-se que este estudo inspire a condução de estudos quase-experimentais, com o objetivo de testar empiricamente a efetividade de programas de intervenção com foco para recursos instrucionais voltados ao desenvolvimento das estratégias de aprendizagem. 


\section{Referências}

Almeida, L. S., \& Guisande, M. A. (2010). Atribuiçóes causais na explicação da aprendizagem escolar. Em E. Boruchovitch, J. A. Bzuneck, \& S. E. R. Guimarães (Orgs.), Motivação para aprender: aplicaçóes no contexto educativo (pp. 145-168). Vozes.

Bahri, A., \& Corebima, A. D. (2015). The contribution of learning motivation and metacognitive skill on cognitive learning outcome of students within different learning strategies. Journal of Baltic Science Education, 14(4), 487-500. https://doi. org/10.33225/jbse/15.14.487

Bardach, L., Oczlon, S., Pietschnig, J., \& Lüftenegger, M. (2019). Has achievement goal theory been right? A meta-analysis of the relation between goal structures and personal achievement goals. Journal of Educational Psychology, 112(6), 1197-1220. https:// doi.org/10.1037/edu0000419

Bernaards, C., Jennrich, R., \& Gilbert, M. P. (2015). Package 'gparotation'. Recuperado de ftp://mirror.cs.uwaterloo.ca/CRAN/web/ packages/GPArotation/GPArotation.pdf

Brasil, Conselho Nacional de Saúde. (2016). Resolução CNS 510, de 07 de abril de 2016. Recuperado de http://conselho.saude.gov.br/ resolucoes/2016/Reso510.pdf

Brasil, Ministério da Educação (2000). Educação Profissional: Referenciais curriculares nacionais da educação profissional de nível técnico. Recuperado de http://portal.mec.gov.br/setec/arquivos/ pdf/introduc.pdf

Bzuneck, J. A., \& Boruchovitch, E. (2019). Motivação de estudantes no ensino superior: Como fortalecê-la? Em L. S. Almeida (Ed.), Estudantes do ensino superior: Desafios e oportunidades (pp. 137-158). ADIPSIEDUC.

Bzuneck, J. A., \& Sales, K. F. S. (2011). Atribuiçóes interpessoais pelo professor e sua relação com emoçóes e motivação do aluno. Psico-USF, 16(3), 307-315. 
Cohen, J. (1992). A power primer. Psychological Bulletin, 112(1), 155-159. https://doi.org/10.1037/0033-2909.112.1.155

Dalbosco, S. N. P., Ferraz, A. S., \& dos Santos, A. A. A. (2018). Metas de realização, autorregulação da aprendizagem e autopercepção de desempenho em universitários. Revista Brasileira de Orientação Profissional, 19(1), 75-84. http://dx.doi.org/1026707/1 984-7270/2019v19n1p75

Dunn, T. J., Baguley, T., \& Brunsden, V. (2014). From alpha to omega: A practical solution to the pervasive problem of internal consistency estimation. British Journal of Psychology, 105(3), 399-412. https://doi.org/10.1111/bjop.12046

Fedre, J. P., \&Vitorino, A. J. R. (2015). Apontamentos das atuais políticas públicas relativas ao ensino profissionalizante no Brasil. Comunicaçóes, 22(1), 159-172. https://doi.org/10.15600/2238-121X/ comunicacoes.v22n1 p159-172

JASP. (2018). Statistical analysis in JASP: A guide for students. Mark A Goss-Sampson.

Jossberger, H., Brand-Gruwel, S., van de Wiel, M. W., \& Boshuizen, H. P. (2019). Exploring Students' Self-Regulated Learning in Vocational Education and Training. Vocations and Learning, 1-28. https://doi.org/10.1007/s12186-019-09232-1

Hulleman, C. S., Schrager, S. M., Bodman, S. M., \& Harackiewicz, J. M. (2010). A meta-analytic review of achievement goal measures: Different labels for the same constructs or different constructs with similar labels. Psychological Bulletin, 136(3), 422-449. https://doi.org/10.1037/a0018947

Marôco, J. (2014). Análise de equaçôes estruturais: Fundamentos teóricos, software \& aplicaçóes. ReportNumber.

Muthén, L. K., \& Muthén, B. O. (2012). Mplus User's Guide: Statistical Analysis with Latent Variables (7th ed.). Muthén \& Muthén.

Pereira, C. P. S., Santos, A. A. A. (2016). Escala de Avaliação de Estratégias de Aprendizagem para o Ensino Profissionalizante. [Relatório Técnico não Publicado]. Universidade São Francisco: Itatiba, SP. 
Pereira, C. P. S., Santos, A. A. A., \& Ferraz, A. S. (2020). Escala de avaliação das estratégias de aprendizagem (ensino profissionalizante): Adaptação e estudos psicométricos. Revista Portuguesa de Educação, 33(1), 75-93. https://doi.org/10.21814/rpe.19127

R Core Team. (2019). R: A language and environment for statistical computing. Vienna, Austria: R Foundation for Statistical Computing. Recuperado de https://www.Rproject.org/

Revelle, W. R. (2017). Psych: Procedures for personality and psychological research. Software. Recuperado de http://cran.r-project.org/web/ packages/psych/psych.pdf

Rothes, A., Lemos, M. S., \& Gonçalves, T. (2017). Motivational profiles of adult learners. Adult Education Quarterly, 67(1), 3-29. https://doi.org/10.1177/0741713616669588

Rozendaal, J. S., Minnaert, A. E. M. G., \& Boekaerts, M. (2003). Motivation and self-regulated learning in secondary vocational education: Information-processing type and gender differences. Learning and Individual Differences, 13(4), 273-289. https://doi. org/10.1016/S1041-6080(03)00016-5

Santos, A. A. A., Alcará, A. R., \& Zenorini, R. D. P. C. (2013). Estudos psicométricos da escala de motivação para a aprendizagem de universitários. Fractal: Revista de Psicologia, 25(3), 531-546. https://doi.org/10.1590/S1984-02922013000300008

Santos, A. A. A., \& Inácio, A. L. M. (2020). Motivação no ensino médio: Metas de realização e estratégias de aprendizagem. Revista Psicologia-Teoria e Prática, 22(2), 338-359.

Santos, A. A. A., \& Mognon, J. F. (2016). Motivation assessment scale for learning in higher education: Validity evidence. Psico-USF, 21(1), 101-110 https://doi.org/10.1590/1413-827120162101 09.

Scacchetti, F. A. P., Oliveira, K. L. D., \& Moreira, A. E. D. C. (2015). Estratégias de aprendizagem no ensino técnico profissional. Psico-USF, 20(3), 433-446. https://doi. org/10.1590/1413-82712015200306 
Scacchetti, F. A. P., Oliveira, K. L., \& Rufini, S. É. (2014). Medida de motivação para aprendizagem no Ensino Técnico Profissional. Avaliação Psicológica, 13(2), 297-305.

Senko, C., \& Dawson, B. (2017). Performance-approach goal effects depend on how they are defined: Meta-analytic evidence from multiple educational outcomes. Journal of Educational Psychology, 109, 574-598. https://doi.org/10.1037/edu0000160

Slaats, A., Lodewijks, H. G., \& van der Sanden, J. M. (1999). Learning styles in secondary vocational education: disciplinary differences. Learning and instruction, 9(5), 475-492. https://doi. org/10.1016/S0959-4752(99)00007-9

van Grinsven, L., \& Tillema, H. (2006). Learning opportunities to support student self-regulation: Comparing different instructional formats. Educational Research, 48(1), 77-91. https://doi. org/10.1080/00131880500498495

Van Yperen, N. W., Elliot, A. J., \& Anseel, F. (2009). The influence of mastery-avoidance goals on performance improvement. European Journal of Social Psychology, 39, 932-943. https://doi. org/10.1002/ejsp.590

Zenorini, R. P. C., \& Santos, A. A. A. (2010). Escala de Metas de Realização como medida da motivação para a aprendizagem. Interamerican Journal of Psychology, 44(2), 291-298. Recuperado de http://www.redalyc.org/ pdf/284/28420641010.pdf

Recibido: 2020-11-08

Revisado: 2021-11-11

Aceptado: 2021-11-24 